\title{
Prevalence of Chronic Diseases among International Travelers Seeking Pretravel Medical Advice in 2018 at Malaga, Spain
}

\author{
Rosa M. Lopez-Gigosos, ${ }^{1,2 \star}$ Marina Segura, ${ }^{2}$ Eloisa Mariscal-Lopez, ${ }^{1}$ Mario Gutierrez-Bedmar, ${ }^{1}$ and Alberto Mariscal ${ }^{1}$ \\ ${ }^{1}$ Department of Public Health and Psychiatry, Faculty of Medicine, University of Malaga, Malaga, Spain; ${ }^{2}$ International Vaccination Center of \\ Malaga, Maritime Port of Malaga, Ministry of Health, Consumption and Social Welfare, Government of Spain, Malaga, Spain
}

\begin{abstract}
Travelers with preexisting diseases or chronic conditions may be more susceptible to travel-related health risks. They may, therefore, require more attention from specialist travel medicine providers. Our objective was to examine a group of international travelers in Malaga, Spain, quantify the proportion of travelers suffering from chronic conditions, and understand the characteristics of this group. A representative sample of travelers requesting pretravel medical advice at one travel clinic were asked about their preexisting chronic conditions and any immunosuppression. Additional demographic variables were used in an analysis of bivariate correlations. We used a binary logistic regression analysis to identify relationships between independent variables (age, gender, type of trip, travel duration, and destination) and the presence or absence of chronic conditions in travelers. Of the sample of 1,196 travelers, $258(21.6 \%)$ reported having preexisting chronic conditions and $72(6 \%)$ had two or more chronic conditions. Twenty-four of the travelers with chronic conditions (9\%) were immunocompromised because of the disease or treatment. The two most common chronic conditions were cardiovascular disease and chronic respiratory conditions (36.8\% and $17.1 \%$, respectively). The chronic condition increased by $6.7 \%$ for every year of increased age. Travelers with chronic conditions are older, travel mainly to visit friends and relatives, and take shorter trips. More than half of travelers visiting (55.8\%) needed more attention from the travel medicine practitioner because of their preexisting chronic conditions, age, or type of travel. Surveillance data based on the population of people traveling would be helpful to provide better advice to travelers.
\end{abstract}

\section{INTRODUCTION}

In 2017, almost 1.4 billion international arrivals (overnight visitors) occurred around the world, and this is expected to increase to almost two billion by $2030 .^{1}$ A large proportion of these trips will be made to countries with considerable health risks, so it is likely that the demand for pretravel medical advice will continue to grow. ${ }^{1}$

The profile of travelers and destinations are constantly changing. In recent years, there have been advances in medical treatment that have improved quality of life for patients with chronic conditions. At the same time, more trips have become available to secure international destinations, which has increased the number of older travelers or those with preexisting medical conditions. It is common to find travelers of all ages, including children, adolescents, and older people, and many have chronic conditions such as heart disease, diabetes, asthma, or arthritis. ${ }^{2-4}$ These travelers require specialized attention from experts in travel medicine, to check their routine medication, and identify possible interactions with treatments or recommended vaccines for the trip. ${ }^{4}$ Some vaccines, such as live-attenuated vaccines, are contraindicated for some patients, and antimalarial chemoprophylaxis can interact with some medications. It is, therefore, necessary to weigh the risks and benefits before providing recommendations. Noncommunicable diseases are the major causes of disability and death globally, including $70 \%$ of all deaths (93\% in Spain), ${ }^{5}$ and tend to become more common with age. ${ }^{5}$ The risk of travel-related diseases is more than doubled in people with underlying medical conditions compared with healthy individuals. ${ }^{6}$ Providing travel medicine

*Address correspondence to Rosa M. Lopez-Gigosos, Department of Public Health and Psychiatry, Faculty of Medicine, University of Malaga, Boulevard Louis Pasteur, 32 29071-Málaga 29071, Spain. E-mail: roslopgig@uma.es advice for patients with chronic conditions requires a personalized plan for vaccines and prophylaxis.

The objective of this study was to identify the proportion of international travelers suffering from chronic conditions in a group of people who sought pretravel medical advice at the International Vaccination Center (IVC) in Malaga and describe their characteristics, including age, gender, duration and type of travel, and destination.

\section{MATERIALS AND METHODS}

This was a prospective, observational, and descriptive study of travelers requesting pretravel medical advice at the IVC in Malaga (South of Spain). Participants were recruited prospectively. To estimate a prevalence of chronic conditions of $20 \%$ with an absolute precision of $\pm 2.5 \%, 909$ participants were required. For the sample size calculation, we used the formula for estimating a proportion on a finite population $(12,000$ travelers per year) with $95 \% \mathrm{Cl}$ and a design effect of 1 . The expected prevalence (20\%) was based on a previous pilot study on 30 participants, and the population was inferred according to a previous study. ${ }^{7}$ During the data collection period (3 months), a total of 3,000 travelers were expected. To achieve the required sample size, we used a systematic random sample with a skip interval of 3 . In total, 11,402 travelers visited the IVC in 2018. One in every two travelers visiting the IVC between September 1 and November 30, 2018 was randomly selected. Travelers returning for a second or subsequent visit during the study period were excluded. Two medical professionals trained in pretravel consultations conducted face-to-face interviews in Spanish, English, or French to complete a questionnaire in a closed electronic system (SISAEX, Madrid, Spain) during the consultation. The questionnaire included information on age, gender, countries visited, travel duration in days, reason for travel (tourism, business, visiting friends and relatives (VFR), or humanitarian cooperation), presence and type of pre-diagnosed chronic 
conditions, and presence and type of immunosuppression. The SISAEX (Foreign Health Information System) is a database management system that collects data on travelers requesting travel-related health information at all IVCs in Spain. Authorization for the handling of the SISAEX data was obtained from the responsible agency at the Spanish Health Ministry. The authors had no access to any identifying personal information. During the face-to-face interview, travelers were informed about the study and asked if they agreed to participate. All travelers expressed their consent for the study and were informed that their data will be used for centralized control procedures and can be used for statistical purposes and health research. For this noninterventional study, no ethics committee approval was required.

Data de-identified were entered anonymously and analyzed using SPSS v25 (SPSS Inc., Chicago, IL). Characteristics of participants were described as means and SD for continuous variables and percentages for categorical variables. Group comparisons were carried out using Student's $t$-test, one-way analysis of variance (ANOVA), or chi-squared test as appropriate. To study the association between chronic conditions and independent variables, we used a multivariate logistic regression model, including potential confounders and those variables that had reached at least a $P<0.20$ in the bivariate analysis. Variables included in the final model were age (years), gender, Africa destination (binary: yes/no), Southeast destination (binary: yes/no), duration of trip (binary: $\leq 30$ days/ $>30$ days), and type of trip as VFR (binary: yes/no). All statistical tests were two-sided, and $P$ values $<0.05$ were considered statistically significant.

\section{RESULTS}

A total of 1,196 travelers (50.3\% female) were included in the study, and $10.5 \%$ of the total number of travelers $(11,402)$ visited the IVC during 2018. The mean age of the travelers was
38 years $(S D=15.75)$, and they traveled mainly as tourists $(N=$ $802,67.1 \%)$ to countries in WHO Southeast Asia Region $(N=$ $404,33.78 \%)$ or the WHO Americas Region ( $N=346,28.93 \%)$ for between 8 and 30 days ( $N=818,68.39 \%)$. Overall, 258 travelers $(21.6 \%)$ had at least one chronic condition. Demographic and travel characteristics of travelers with and without disease or chronic condition are summarized in Table 1.

Travelers aged 65 years or older made more often $(83.4 \%$, 95\% $\mathrm{Cl} 73.6-90.6)$ shorter trips ( $\leq 30$ days) than travelers aged less than 65 years $(73.5 \%, 95 \% \mathrm{Cl} 70.8-76.5)$, although these differences were borderline-significant $(P=0.08)$. On the other hand, travelers aged 65 years or older made trips mainly as tourists $(85.7 \%, 95 \% \mathrm{Cl} 76.4-92.4$ versus $65.5 \%, 95 \% \mathrm{Cl}$ 62.6-68.3; $P<0.001)$. Two hundred and thirty-six subjects (19.7\%) were VFR travelers, of whom $65.3 \%$ and $29.3 \%$ went to the Americas and Africa, respectively. Of those younger than 16 years $(N=87), 62.1 \%$ were VFR who traveled to the Americas (72.2\%) and Africa (27.8\%).

The mean age of travelers with chronic conditions was higher than those who did not report chronic conditions (49.4 years, $S D=16.3$ versus 35.9 years, $S D=14.1 ; P<0.001)$. The chronic conditions were more frequent in traveler 65 years or older $(66.7 \%, 95 \% \mathrm{Cl} 55.5-76.6)$ compared with those between 46 and 64 years old $(37.1 \%, 95 \% \mathrm{Cl} 31.1-43.4)$ or younger than $45(12.7 \%, 95 \% \mathrm{Cl} 10.6-15.1)(P<0.001)$. Comorbidity was also frequent, and 72 of travelers with chronic conditions (27.9\%) reported having two $(22.1 \%)$ and three or more $(5.8 \%)$ diseases. The mean age of travelers with one, two, and three or more chronic conditions was 46.1 (95\% Cl 43.7-48.5), 57.4 (95\% Cl 45.8-68.9), and 60.5 years $(95 \%$ Cl 55.2-65.8) $(P<0.001)$, respectively. There were no significant differences in gender, duration, or type of trip between travelers with and without chronic diseases by bivariate analysis (Table 1). Nevertheless, more men than women $(33 / 23)$ older than 65 years reported chronic conditions $(P=$ 0.008). The proportion of travelers with chronic conditions was

TABLE 1

Chronic conditions and demographic and travel characteristics of travelers

\begin{tabular}{|c|c|c|c|}
\hline $\begin{array}{c}\text { Demographic and travel } \\
\text { characteristics }\end{array}$ & $\begin{array}{l}\text { Travelers with disease or chronic } \\
\text { condition }(N=258) \%\end{array}$ & $\begin{array}{l}\text { Travelers without disease or chronic } \\
\text { condition }(N=938) \%\end{array}$ & $P$-value \\
\hline \multicolumn{4}{|l|}{ Gender } \\
\hline Male & 125 (48.4) & $470(50.1)$ & \multirow[t]{2}{*}{0.344} \\
\hline Female & 133 (51.6) & 468 (49.9) & \\
\hline \multicolumn{4}{|l|}{ Age (years) } \\
\hline$\leq 15$ & $6(2.3)$ & $81(8.6)$ & \multirow[t]{3}{*}{$<0.001$} \\
\hline $16-64$ & $196(76.0)$ & $829(88.4)$ & \\
\hline$\geq 65$ & $56(21.7)$ & $28(3.0)$ & \\
\hline \multicolumn{4}{|l|}{ Type of trip } \\
\hline Tourist & $169(65.5)$ & $633(67.5)$ & \multirow[t]{4}{*}{0.587} \\
\hline Business & $20(7.8)$ & $91(9.7)$ & \\
\hline Cooperation & $11(4.3)$ & $36(3.8)$ & \\
\hline VFR & $58(22.5)$ & $178(19.0)$ & \\
\hline \multicolumn{4}{|l|}{ Duration (days) } \\
\hline$<8$ & $14(5.4)$ & $55(5.9)$ & \multirow[t]{3}{*}{0.265} \\
\hline $8-30$ & $187(72.5)$ & $631(67.3)$ & \\
\hline$>30$ & 57 (22.1) & 252 (26.9) & \\
\hline \multicolumn{4}{|l|}{ WHO region visited* } \\
\hline Africa & $68(26.4)$ & $206(22.0)$ & \multirow[t]{6}{*}{0.024} \\
\hline Americas & 78 (30.2) & $268(28.6)$ & \\
\hline Southeast Asia & $68(26.4)$ & $336(35.8)$ & \\
\hline Europe & $4(1.6)$ & $5(0.5)$ & \\
\hline Eastern Mediterranean & $7(2.7)$ & $13(1.4)$ & \\
\hline Western Pacific & $33(12.8)$ & $110(11.7)$ & \\
\hline
\end{tabular}

* Travel destinations grouped into $\mathrm{WHO}$ geographical regions. 
TABLE 2

Preexisting health conditions among the 258 travelers who reported chronic condition

\begin{tabular}{|c|c|c|c|}
\hline \multirow[b]{3}{*}{ Clinical conditions } & \multicolumn{3}{|c|}{ Age (years) } \\
\hline & $<64$ & $\geq 65$ & \\
\hline & $N=202(\%)$ & $N=56(\%)$ & $P$-value \\
\hline Chronic disease & & & $<0.001$ \\
\hline Cardiovascular & $65(32.2)$ & $45(80.4)$ & \\
\hline Chronic respiratory & $46(22.8)$ & $2(3.6)$ & \\
\hline Endocrine diseases* & $28(13.9)$ & 7 (12.5) & \\
\hline Joint pathology & $28(13.9)$ & $12(21.5)$ & \\
\hline Psychiatric pathology & $22(10.9)$ & $10(17.0)$ & \\
\hline Type 1 and 2 diabetes & $19(9.4)$ & $9(16.1)$ & \\
\hline Neurological pathology & $18(8.9)$ & $1(1.8)$ & \\
\hline Cancer & $12(5.9)$ & $0(0.0)$ & \\
\hline Othert & $16(7.9)$ & $2(3.6)$ & \\
\hline Immunocompromised & & & 0.008 \\
\hline $\begin{array}{l}\text { Congenital/acquired } \\
\text { immunosuppression disease }\end{array}$ & $5(2.5)$ & $3(5.4)$ & \\
\hline Corticosteroids/cytostatic treatment & $11(5.4)$ & $2(3.6)$ & \\
\hline Monoclonal/polyclonal Ab treatment & $2(1.0)$ & $1(1.8)$ & \\
\hline
\end{tabular}

† This includes aplastic anemia (1), chronic liver disease (5), chronic renal failure (6), morbid obesity (1), and psoriasis (5).

lower in those visiting Southeast Asia (16.8\%, 95\% Cl 13.3-20.8) and higher among those going to Western Mediterranean (35.0\%, 95\% Cl 15.4-59.2, $P=0.038)$.

The most frequently seen chronic conditions were cardiovascular disease (42.6\%) (Table 2). The mean age was higher for travelers with cardiovascular disease $(58.3$ years, SD = 13.0) and diabetes $(54.6$ years, $S D=11.0)$ than chronic respiratory disease $(38.8$ years, $S D=16.1)$ or cancer $(45.2$ years, $\mathrm{SD}=14.7)$.

During the consultation, 24 travelers with chronic conditions also reported that they were immunosuppressed because of a disease or treatment. This was $9.3 \%$ of travelers with chronic conditions and $2 \%$ of all travelers.

The proportion of travelers immunocompromised by medication and/or disease increased with age, from 1.6\% (95\% Cl $1.0-2.5)$ for travelers younger than 65 years to $7.1 \%(95 \% \mathrm{Cl}$ 2.7-14.9) for older travelers $(P<0.001)$. There were no significant differences in the frequency of immunosuppression by gender or in the duration of trip between travelers with and without chronic conditions, but only three of the 24 immunosuppressed travelers made trips of more than 30 days.

A binary logistic regression analysis was used to predict the presence or absence of chronic disease in travelers visiting the IVC, and the relationship with independent variables (age, gender, type of trip, travel duration, and destination). The final model selected included age, gender, a destination in Southeast Asia or Africa, the duration of the trip, and trips to visit friends or relatives (Table 3). The model was significant and $81.8 \%$ of the cases were correctly classified. Of the variables introduced in the model, age was a significant predictor $(P<0.001)$. For every year of increased age, the risk of having a chronic condition increased by $6.7 \%$. It could be inferred from the model that travelers with chronic conditions are older, travel mainly as VFR, take shorter trips, and are less likely to visit Southeast Asia.

\section{DISCUSSION}

Our study investigated the number of travelers with preexisting medical conditions in a representative sample of travelers visiting an IVC in southern Spain. The mean age of the traveler (38 years) was 7 years higher than in another study in Spain between 1999 and $2004^{8}$ but similar (37 years) to that found in more recent studies (2009-2011) in Marseilles and the United States. ${ }^{3,9}$ However, there were some differences when comparing the most frequent travel destinations in the traveling population studied and those found in other cities in Europe and the United States. ${ }^{3,9,10}$ The high number of travelers (42.4\%) to Southeast Asia or the Western Pacific reflects an international positive trend previously described in recent years. ${ }^{7,11,12}$ On the other hand, the high number of travelers to the Americas (28.9\%), especially to South America, reflects historical and cultural links and language. Almost all travelers to the Americas in this study were going to countries in South or Central America because travelers to Canada or the United States generally do not need specialized travel assistance.

Most travelers were healthy and required a standard pre-trip consultation that includes a brief medical background check, personal risk assessment, the need for vaccination or chemoprophylaxis for malaria, and information about protection against insects, diarrhea, problems with water and food, or altitude. ${ }^{13,14}$ Other travelers, however, require more time in their pre-trip consultations. In this study, we included in this group travelers with chronic diseases $(21.6 \%)$, VFR travelers (19.7\%), people aged 65 years and older (7\%), and children

TABLE 3

Binary logistic compliance model

\begin{tabular}{lcc}
\hline \multicolumn{1}{c}{ Variables } & Odds ratio & $95 \% \mathrm{Cl}$ \\
\hline Age (years) & 1.067 & $1.056-1.078$ \\
Gender (male*) & 0.839 & $0.619-1.137$ \\
Destination $\dagger$ & & \\
$\quad$ Africa & 0.928 & $0.637-1.352$ \\
$\quad$ Southeast Asia & 0.648 & $0.441-0.953$ \\
Duration of trip $\dagger$ & 0.797 & $0.544-1.169$ \\
Visits to friends and relatives $†$ & 1.515 & $1.011-2.272$ \\
$\quad$ Odds ratios and 95\% Cls for associations between preexisting health conditions and \\
variables selected. \\
$\quad{ }^{*}$ Reference category. \\
$\quad+$ Variables were codified as follows: Africa destination (binary: yes/no), Southeast \\
destination (binary: yes/no), duration of trip (binary: $\leq 30$ days/> 30 days), and type of trip as \\
VFR (binary: yes/no).
\end{tabular}


aged 15 years or younger $(7.4 \%)$. We found that $42.6 \%$ of travelers had special requirements, slightly more than the percentage found by Aubry (36\%) or Bühler (35.3\%). ${ }^{2,3}$ In our study, the number of VFR travelers was almost $20 \%$, which was higher than that found (8-16.5\%) in other studies from the European Union. ${ }^{2,3,8}$ The main travel destinations of VFR (94.6\% to America and Africa) usually involve immigrants from South or Central America and Africa, and reflect the high immigrant population from both those regions in Malaga. The reason for the increase could be because of recent improvements in the economic situation because economic crises have clear effects on the number of travelers each year. ${ }^{7,15}$

As expected, the frequency of chronic conditions among travelers increased with age, ranging from $6.7 \%$ in children younger than 16 years to $66.7 \%$ in those older than 65 years. These data are similar to those found by Aubry et al. ${ }^{3}$ in children younger than 16 years, although the number of travelers older than 60 years with chronic conditions was significantly higher in our study than in Marseilles (27\%), Zurich (about $40 \%$ ), or Galway (56\%). $3,16,17$ This may be partially because the mean age of the travelers visiting the IVC was higher than in these other studies, and therefore, the proportion with chronic conditions was also greater. Previous studies have found that the mean age of travelers with chronic conditions was $32,{ }^{17}$ $39,{ }^{4}$ and $47,{ }^{18}$ compared with 49 years in our study. These differences may be explained by the methodology and date of the study or the demographic composition of the populations.

In total, $9.2 \%$ of the travelers visiting the IVC and $53.6 \%$ of those of 65 years or more had cardiovascular disease. Previous studies have found levels of cardiovascular disease among travelers ranging from $1.8 \%$ to $12.8 \% .^{2,3,17}$ These differences, as with other chronic conditions included in the study, are the result of local demographic differences in the populations studied and the methodology used in each study. We asked travelers about their chronic conditions using the WHO chronic disease classification, ${ }^{5}$ but other studies have used alternatives that make it difficult to directly compare the results. Factors or conditions known to interact with the most common pretravel prescriptions for malaria, yellow fever, or diarrhea were included in the study by Van de Winkel et al., ${ }^{4}$ Valim et al., ${ }^{19}$ Bica et al. ${ }^{20}$ However, Aubry et al., ${ }^{3}$ in their prospective study on travelers visiting pretravel medical consultations, collected more detailed information about chronic medical conditions. They excluded pilgrims to Mecca in the Haj and people like the crew of planes and ships. ${ }^{3}$ In a similar study by Han et al., ${ }^{17}$ common medical conditions were included, including aspects with particular relevance for travel vaccines such as immunosuppression and egg allergies. They included only travelers with a previous medical history and/or those taking prescribed medications for chronic or ongoing diseases. ${ }^{17}$

The improvement in life expectancy and treatment of chronic diseases provides opportunities for travel by patients whose immune system has been suppressed by drugs or disease. These travelers, estimated to be between $0.7 \%$ and $4 \%$ of travelers, ${ }^{2,3,10,17}$ were well represented in our study, with 24 individuals ( $2 \%$ of travelers), and require specialized support from medical experts in travel medicine. $4,21,22$

This study has provided valuable information about the characteristics of international travelers. However, it is limited by not having access to medical records containing information about chronic conditions, apart from the information obtained by doctors at the IVC during an interview with the traveler.
However, the interviews were carried out by specialist doctors and, therefore, provide more information than a simple questionnaire about medical conditions. Generally, travelers do not refuse to provide detailed information when doctors ask about their medication because they know that some medication may interact with some recommended prophylaxis. This study included only travelers visiting the IVC, so those who did not seek medical advice or consulted other sources such as general practitioners were not included. The period studied was also limited to 3 months, between September and November, so it could have been influenced by seasonal effects. The percentage of travelers with a chronic condition could be lower during common holiday periods in the Northern Hemisphere (July-August), in which younger and healthier travelers can travel. However, our objective was to find the most common chronic conditions among travelers visiting our IVC.

\section{CONCLUSION}

Medical consultations for travelers who require special assistance because of a chronic condition, ongoing or planned pregnancy, age (older or very young), and VFR travelers require particular attention to adapt vaccination and pretravel medical advice to this vulnerable population. Our study, like most studies in specialized travel clinics, is not representative of the entire traveling population, but it does represent most travelers traveling to countries where there are specific risks, such as yellow fever, malaria, Zika, Japanese encephalitis, and other tropical diseases. The high number of people with special requirements traveling to high-risk areas means that travel medicine provision requires ongoing training on both to the health status of travelers and the treatments they may be receiving. At the same time, it is also important to train doctors such as pediatricians, geriatricians, internists, general practitioners, and specialist physicians caring for immunocompromised people in travel medicine.

Received September 21, 2019. Accepted for publication December 5, 2019.

Published online January 13, 2020.

Acknowledgments: We thank Melissa Leffler, MBA, from the Edanz Group (www.edanzediting.com/ac) for editing a draft of this manuscript.

Authors' addresses: Rosa M. Lopez-Gigosos, Eloisa Mariscal-Lopez, Mario Gutierrez-Bedmar, and Alberto Mariscal, Department of Public Health and Psychiatry, Faculty of Medicine, University of Malaga, Malaga, Spain, E-mails: roslopgig@uma.es, emariscal@uma.es, bedmar@uma.es, and mariscal@uma.es. Marina Segura, International Vaccination Center of Malaga, Maritime Port of Malaga, Ministry of Health, Consumption and Social Welfare, Government of Spain, Malaga, Spain, E-mail: marina.segura@correo.gob.es.

\section{REFERENCES}

1. World Tourism Organization, 2018. UNWTO Tourism Highlights, 2018 Edition. Available at: https://www.e-unwto.org/doi/pdf/10. 18111/9789284419876. Accessed September 10, 2019.

2. Bühler S, Rüegg R, Steffen R, Hatz C, Jaeger VK, 2014. A profile of travelers - an analysis from a large Swiss travel clinic. J Travel Med 21: 324-331.

3. Aubry C, Gaudart J, Gaillard C, Delmont J, Parola P, Brouqui P, Gautret $P, 2012$. Demographics, health and travel characteristics of international travellers at a pre-travel clinic in Marseille, France. Travel Med Infect Dis 10: 247-256. 
4. Van De Winkel K, den Daele A, Van Gompel A, den Ende J, 2007. Factors influencing standard pretravel health advice-a study in Belgium. J Travel Med 14: 288-296.

5. WHO, 2018. Noncommunicable Diseases Country Profiles 2018. NCD Ctry Profiles 2018. Available at: www.who.int/nmh/ publications/ncd-profiles-2018/en/. Accessed September 10, 2019.

6. Gudmundsson A, Stevenson JM, Petrovic M, Somers A, Onder G, Callens S, van der Cammen TJM, 2016. Challenges and risks for older travellers with multimorbidity: focus on pharmacotherapy. Eur Geriatr Med 7: 407-410.

7. Segura M, Lopez-Gigosos R, Mariscal-Lopez E, GutierrezBedmar M, Mariscal A, 2019. Trends in the travelers' demand for pre-travel medical advice at a Spanish International Vaccination Center between 2000 and 2017. PLoS One 14: e0217588.

8. Valerio L, Martínez O, Sabrià M, Esteve M, Urbiztondo L, Roca C, 2005. High-risk travel abroad overtook low-risk travel from 1999 to 2004: characterization and trends in 2,622 Spanish travelers. J Travel Med 12: 327-331.

9. Larocque RC et al., 2012. Global TravEpiNet: a national consortium of clinics providing care to international travelers-analysis of demographic characteristics, travel destinations, and pretravel healthcare of high-risk US international travelers, 2009-2011. Clin Infect Dis 54: 455-462.

10. Schwartz BS, Rosen J, Han PV, Hynes NA, Hagmann SH, Rao SR, Jentes ES, Ryan ET, LaRocque RC, Consortium TGT, 2015. Immunocompromised travelers: demographic characteristics, travel destinations, and pretravel health care from the U.S. Global TravEpiNet Consortium. Am J Trop Med Hyg 93: 1110-1116.

11. Angelin $M$, Evengård $B, P a l m g r e n ~ H, 2011$. Travel and vaccination patterns: a report from a travel medicine clinic in northern Sweden. Scand J Infect Dis 43: 714-720.

12. Goesch JN, Simons De Fanti A, Béchet S, Consigny P-H, 2010. Comparison of knowledge on travel related health risks and their prevention among humanitarian aid workers and other travellers consulting at the Institut Pasteur travel clinic in Paris, France. Travel Med Infect Dis 8: 364-372.

13. Rozenberg G, Petersiel N, Korytny A, Bishop B, Mousa A, Fried C, Maister A, Neuberger A, 2019. Standard pre-travel consultation versus shorter consultation combined with smartphone support: a randomized controlled trial. J Travel Med 26: taz025.

14. Freedman DO, Chen LH, Kozarsky PE, 2016. Medical considerations before international travel. N Engl J Med 375: 247-260.

15. Cró S, Martins AM, 2017. Structural breaks in international tourism demand: are they caused by crises or disasters? Tour Manag 63: 3-9.

16. Jaeger VK, Tschudi N, Rüegg R, Hatz C, Bühler S, 2015. The elderly, the young and the pregnant traveler - A retrospective data analysis from a large Swiss Travel Center with a special focus on malaria prophylaxis and yellow fever vaccination. Travel Med Infect Dis 13: 475-484.

17. Han CTJ, Flaherty G, 2015. Profile of travelers with preexisting medical conditions attending a specialist travel medicine clinic in Ireland. J Travel Med 22: 312-317.

18. Hochberg NS et al., 2013. International travel by persons with medical comorbidities: understanding risks and providing advice. Mayo Clin Proc 88: 1231-1240.

19. Valim V et al., 2019. Cardiovascular event and risk factors in primary Sjögren's syndrome from latin-american cohort. Ann Rheum Dis 78 (Suppl 2): 2110.

20. Bica B, Da Silva LP, Pileggi GS, 2019. Effects and safety of the yellow fever vaccine 17DD in patients with immunomediated rheumatic diseases. Ann Rheum Dis 78 (Suppl 2): 2059-2060.

21. Tan EM, Marcelin JR, Virk A, 2019. Pre-travel counseling for immunocompromised travelers: a 12-year single-center retrospective review. Infect Dis Health 24: 13-22.

22. Askling HH, Dalm VA, 2014. The medically immunocompromised adult traveler and pre-travel counseling: status quo 2014. Travel Med Infect Dis 12: 219-228. 